\title{
A Remote Direct Sequence Spread Spectrum Communications Lab Utilising the Emona DATEx
}

\author{
http://dx.doi.org/10.3991/ijoe.v8iS4.2187 \\ Helen Kyomugisha, Tom Kigezi, Cosmas Mwikirize, Roseline Akol, Doreen Orishaba, Michael Kyesswa \\ Makerere University, Kampala- Uganda
}

\begin{abstract}
Remote labs have become popular learning aids due to their versatility and considerable ease of utilisation as compared to their physical counterparts. At Makerere University, the remote labs are based on the standard Massachusetts Institute of Technology (MIT) iLabs Shared Architecture (ISA) - a scalable and generic platform. Presented in this paper is such a lab, addressing the key practical aspects of Direct Sequence Spread Spectrum (DSSS) communication. The lab is built on the National Instruments Educational Laboratory Virtual Instrumentation Suite (NI ELVIS) with the Emona Digital and Analog Telecommunications Experimenter (DATEx) add-on board. It also incorporates switching hardware. The lab facilitates real-time control of the equipment, with users able to set, manipulate and observe signal parameters in both the frequency and the time domains. Simulation and data Acquisition modes of the experiment are supported to provide a richer learning experience.
\end{abstract}

Index Terms-Direct Sequence Spread Spectrum, Emona DATEx, Interactive iLabs Shared Architecture, LabVIEW

\section{INTRODUCTION}

Wireless communication is by any measure the fastest growing segment of the telecommunications industry [1]. Early development of wireless technologies was inhibited by several factors, including spectral capacity limits, propagation effects such as multipath, and the need for asynchronous access [2]. Even more critical today is the security aspect of wireless communications, a subject of growing concern for many individuals and organisations.

An ingenious solution to these challenges was realised in adoption of spread spectrum communications. Spreadspectrum techniques are a means of signal transmission in which the signal occupies a bandwidth in excess of the minimum bandwidth necessary to send the information. The band spread is accomplished by means of a code which is independent of the data, and synchronized reception with the code at the receiver is used for de-spreading and subsequent data recovery. Popular applications include Code Division Multiple Access (CDMA) cellulartelephony networks, Global Positioning Systems, and Bluetooth [3].

Two main types of spread spectrum techniques exist; Direct Sequence Spread Spectrum (DSSS) and Frequency Hopping Spread Spectrum (FHSS). Specifically, DSSS multiplies the data being transmitted by a "noise" signal, typically a pseudorandom number (PN) sequence of 1 and -1 values, at a frequency much higher than that of the original signal. This consequentially makes DSSS signals noise-like, making them hard to detect, intercept or demodulate. Furthermore, they are harder to jam (interfere with) and because they are so wide, the signals are transmitted at a much lower spectral power density than narrowband signals [4].

Owing to the exceptional qualities and rapid adoption of spread spectrum, it is vital that students pursuing Electrical, Telecommunications and Computer Engineering disciplines get versed with this key technology, through experimentation. Makerere University (MAK) has adopted the scalable MIT iLabs Shared Architecture (ISA) as a platform for development and deployment of remote shared laboratories (iLabs). These have helped to mitigate challenges arising from the scarcity of laboratory equipment vis-à-vis the skyrocketing student numbers. iLabs have been used to support several courses in the curricula of the Bachelors of Science in Electrical, Telecommunications and Computer Engineering Programmes over the past seven years.[5]-[7].

The iLabs at MAK utilise the National Instruments Educational Laboratory Virtual Instrumentation Suite (NI ELVIS) with a host of add-on boards, supported by the Laboratory Virtual Instrumentation Engineering Workbench (LabVIEW) graphical programming language. One such board is the Emona Digital Analog Telecommunications Experimenter (DATEx) which uses a block diagram model approach to implement a very wide range of telecommunications experiments [8]. Using its adders, multipliers, noise generators, sequence generators, amplifiers and a tuneable low-pass filer, the principles of DSSS communication can be demonstrated.

The user interface to the DSSS lab is a LabVIEW Virtual Instrument (VI), on which interactive controls for the DATEx are exposed. Remote access to the VI is implemented within the interactive ISA [9].

\section{IMPLEMENTATION}

\section{A. Lab Equipment}

The principal hardware for the DSSS lab is the NI ELVIS/Emona DATEx combination. To alternate different circuit configurations on the DATEx, the NI Signal Conditioning eXtensions for Instrumentation (SCXI) 1169 switch is integrated into the physical architecture. A portable data acquisition device, the NI myDAQ [10], is used to provide four extra oscilloscope channels, necessary for viewing the experiment data. The hardware is shown in Figure 1. 


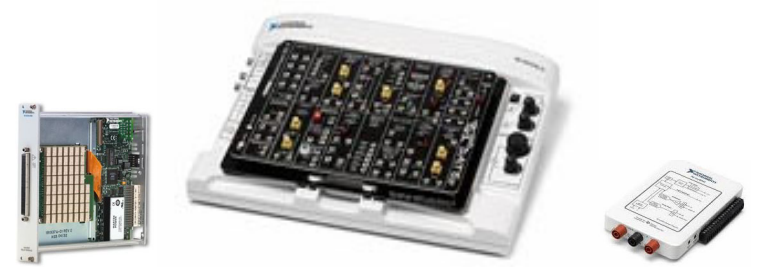

Figure 1. The NI SCXI-1169, NI ELVIS /Emona DATEx Bundle and the NI myDAQ

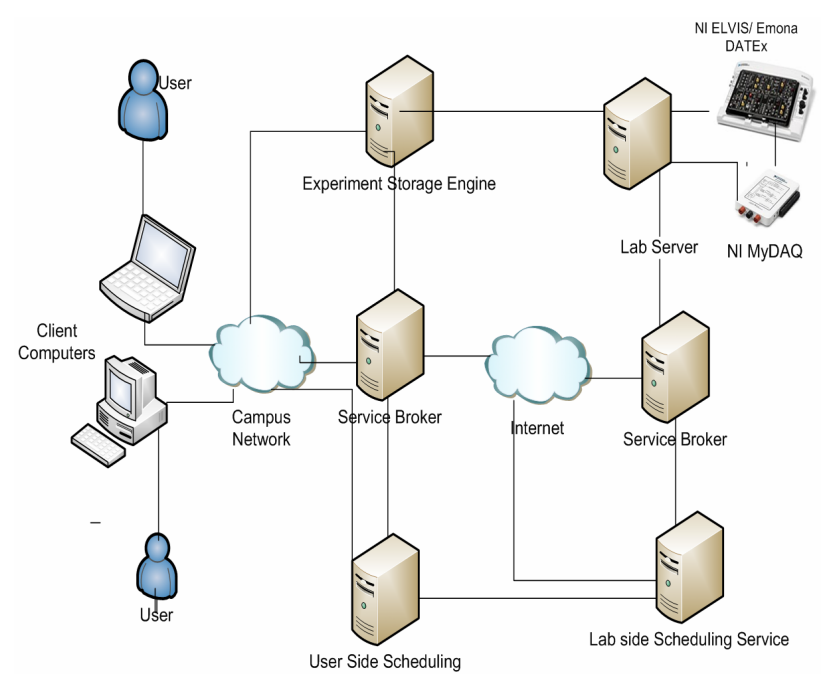

Figure 2. System Context Diagram

The SCXI-1169 switch module is equipped with up to 100 Single-Pole-Single-Throw (SPST) mechanical relay switches [11] but only 7 of these were used for the DSSS application. It is inserted into a chassis (NI SCXI-1000), the box containing the circuitry for powering, fanning and interfacing with the computer hosting the hardware. The system context diagram is shown in Figure 2, illustrating how the hardware is related to the shared lab services within the interactive ISA.

\section{B. Software Architecture and Implementation}

The DSSS circuit is implemented using the block diagram approach shown in Figure 3. The circuit algorithm is easily transferable to the hardware implementation based on a combination of the DATEx modules as shown in Figure 4.

To implement the DSSS remote Lab on the DATEx, one of the considerations was to give the student as much flexibility as possible. Hence, as many DATEx tuneable components as possible were used, including the signal gain and low-pass filter cut-off. It was Emona's polymorphic DATEx VI that was configured appropriately for all the DATEx tuneable blocks while the NI Express VIs (high level control VIs) were used for the NI ELVIS components - the functional generator and the oscilloscope.

Due to the necessity of observing both time and frequency domains, there was a need for a second graph to display the Power Spectrum of the relevant signals. Frequency domain plots of the signals were generated using one of LabVIEW's numerous Fast Fourier Transform (FFT) Power Spectrum VIs. This was the only signal processing technique implemented by LabVIEW for the data acquisition mode of the experiment since no express VI for its Dynamic Signal Analyzer is provided.

The experiment VI supports both simulation and data acquisition modes, which complement each other in enabling the user to compare real and ideal DSSS communication scenarios. The simulation processes a computer generated signal through a software algorithm, producing the DSSS waveforms. The data acquisition mode on the other hand utilizes the DATEx to manipulate a real signal from a functional generator, in which case LabVIEW simply provides the circuit control and experiment data display.

For easier maintenance and code scalability to accommodate future expansion of the lab, the DSSS experiment VI contained three while loops running the simulation, data acquisition and switching hardware in parallel. One STOP control would thus be used to simultaneously end their execution.

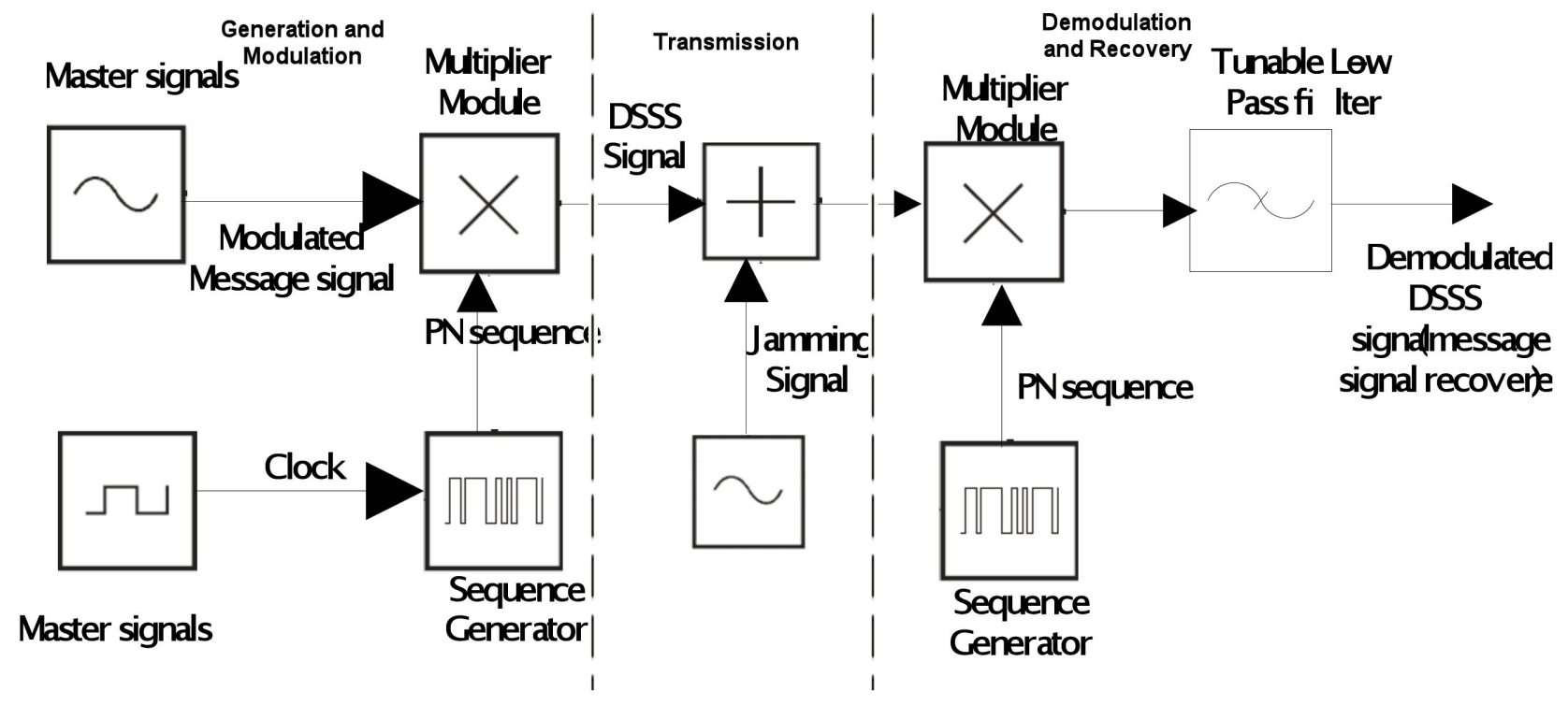

Figure 3. DSSS Block Diagram 


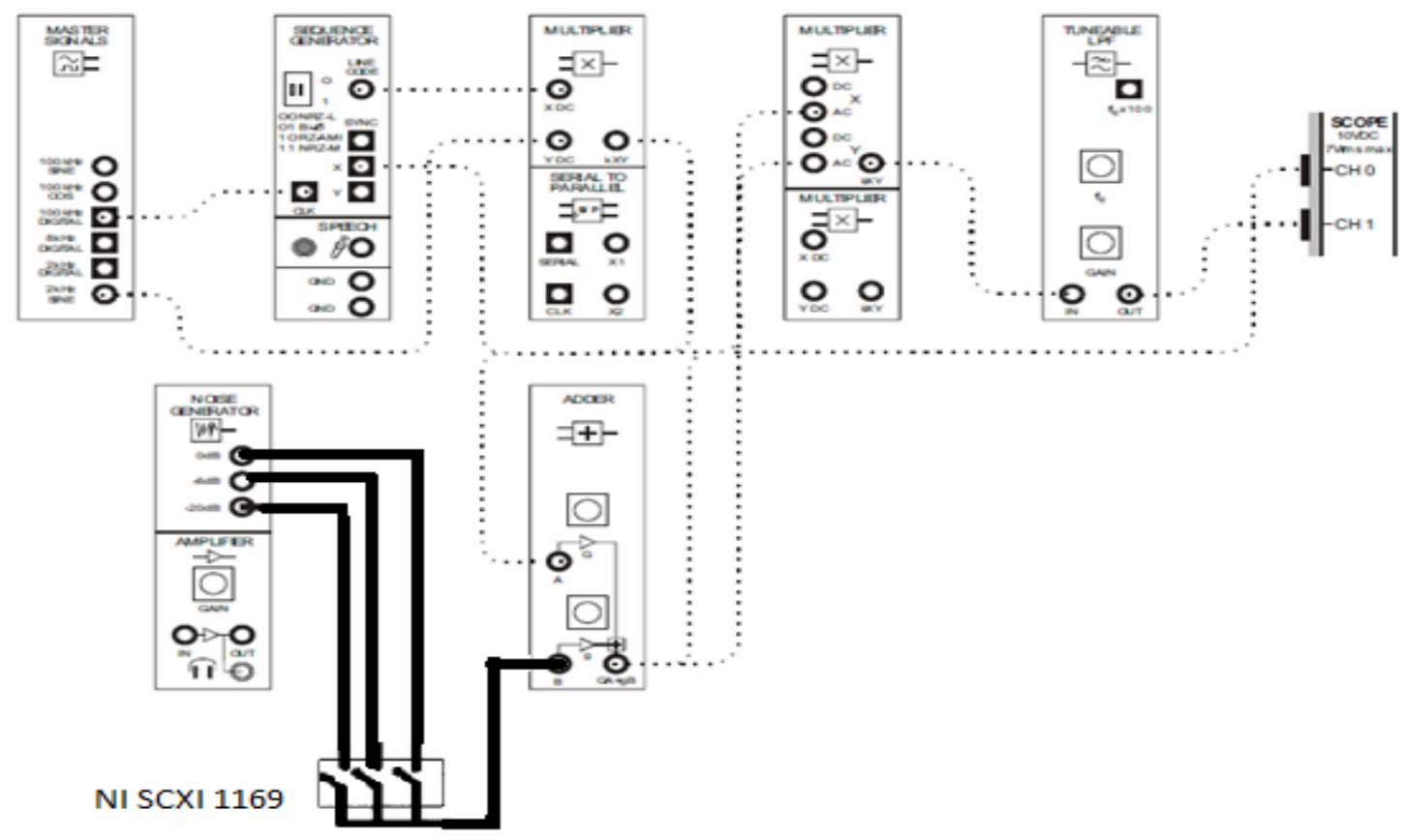

Figure 4. DATEx Wiring Diagram

\section{USER EXPERIENCE}

\section{A. Lab Functionality}

The DSSS lab involves three key stages: Generation \& Modulation of information signal, DSSS signal transmission, and Demodulation and Recovery of information signal. The lab user interface allows interactive variation of virtual hardware controls to achieve each of these stages. Input signal frequency, noise amplitude and PN sequence length are among the variable parameters exposed for user variation. Their effect on the signals under investigation in both time and frequency domains is availed instantly on graphical displays. The modular interaction with the lab is summarised in Figure 5.

\section{B. User Interface Design}

LabVIEW's virtual control elements including knobs, switches, buttons and levers coupled with its indicators such as graphs and LED lights were used to avail a rich and fully interactive front panel which would double as a user interface within the interactive ISA.

Tabbed navigation was incorporated to allow for easy access, control and comparison of the simulation and data acquisition experiment modes. The user was also availed with an ON/OFF switch that allows activation and deactivation of either experiment mode, potentially saving computational resources on less endowed lab server systems.

All observed experiment data is relayed in graphical form to emphasize a holistic interpretation of the experiment at a glance. Strong considerations were made in creating a highly intuitive user interface, thereby appropriately sectioning the controls and graphs according to three key DSSS experiment stages.

\section{EXPERIMENT DYNAMICS}

A typical experiment involves user login, selection of the experiment to be conducted and request for a reserva- tion from the Service Broker. Exclusive access to the lab is granted to the user during his reservation time. With the experiment VI loaded in the user's web browser, the user can then start the experiment by activating either one of the experiment modes. The adjustments and subsequent observations can then be made in the three experiment key stages.

\section{A. Generation \& Modulation of Information Signal}

With an active user interface, the experiment commences with the user setting the information signal type (sine, square, saw-tooth), amplitude and frequency. The desired length of the modulating PN sequence is then selected. Observations are made on these two signals in time and frequency domain, comparing their bandwidths and spectral amplitude levels. The PN sequence power spectrum should stand out as flat or 'noise like' compared to the narrowband information signal. The combination of these two signals produces a DSSS signal.

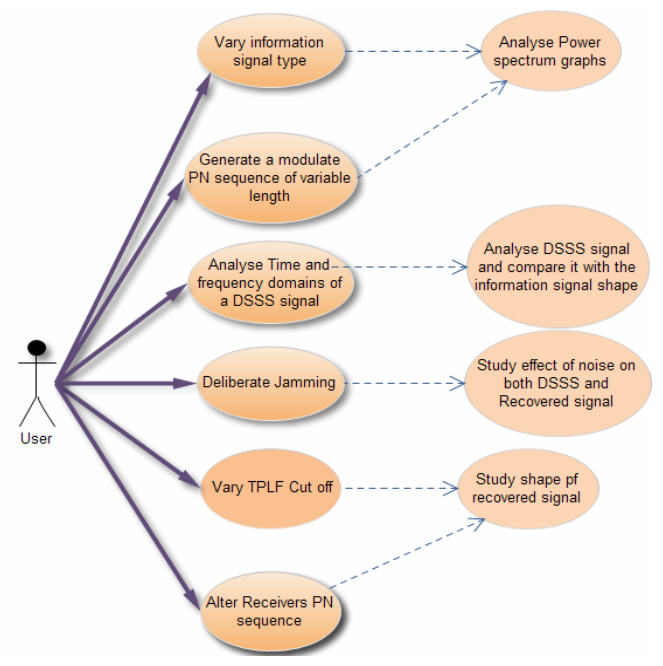

Figure 5. Modular Interaction with the DSSS Lab 


\section{B. DSSS Signal Transmission}

This entails time and frequency domain analysis of the DSSS signal. It should be observed that it adopts the flatness of the modulating PN sequence. The lab user then deliberately jams the signal by adding different signal jamming types (singleton and broadband jamming) of various powers to the transmitted DSSS signal. Its distortion in time domain and the resultant effect on its power spectrum are carefully analyzed.

\section{Demodulation and Recovery of Information Signal}

This includes alteration of receiver's demodulating PN sequence and studying the effect that these variations have on the recovered signal. To correctly recover the signal the user ensures that the same modulating PN sequence is used for demodulation. Gradual adjustment of the tuneable low-pass filter cut-off must also be made until the recovered signal shape is similar to the transmitted signal shape in time and frequency domains. The jamming effects on the recovered signal may then be studied and the extent of DSSS resistance to intentional interference is noted after extreme jamming attempts.

In both experiment modes, the user can download experiment data and analyse it with third-party computer applications such as Microsoft Excel. The simulation and experiment modes of the running lab as accessed through a web browser are shown in Figures 6 and 7 respectively.

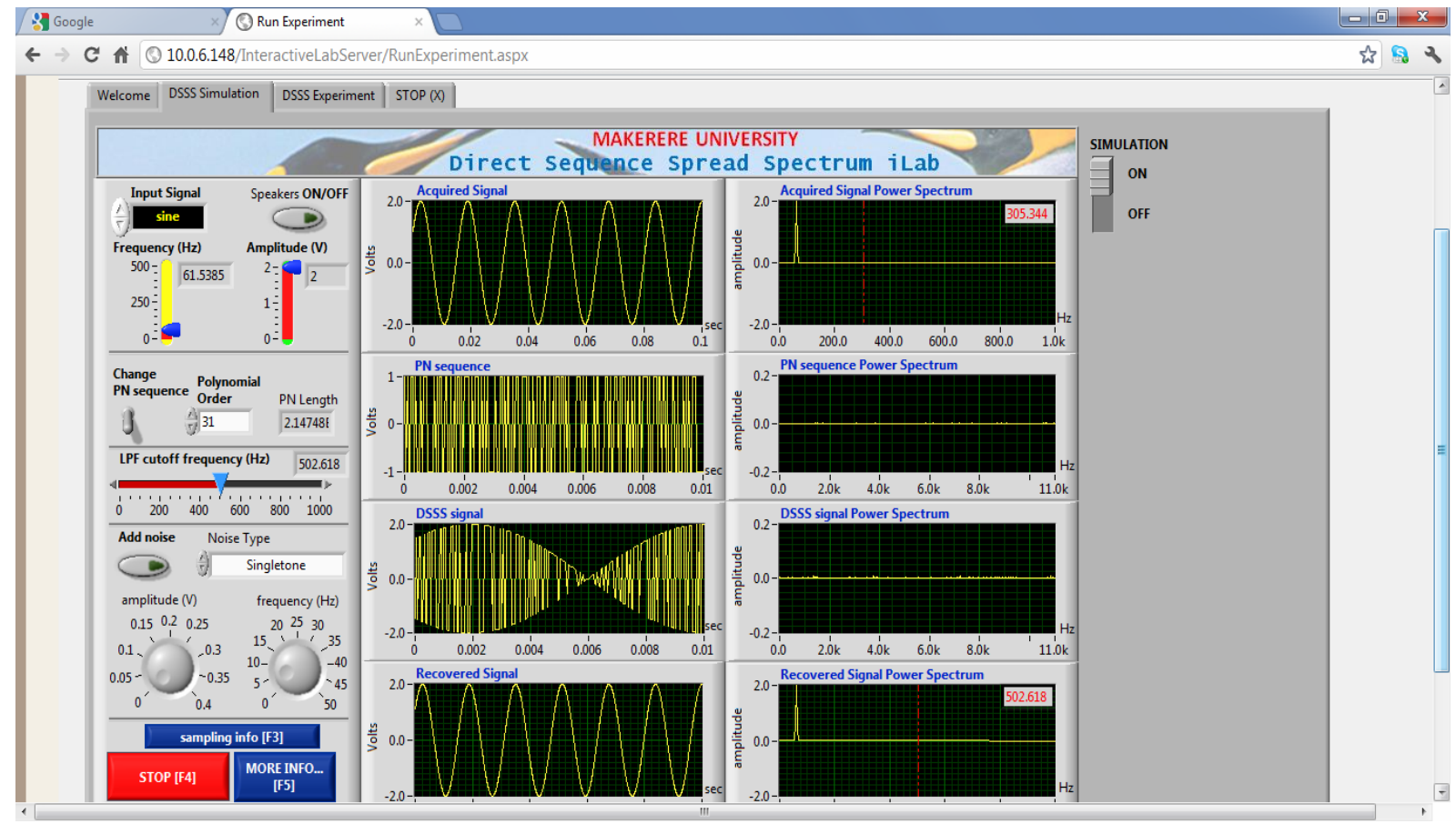

Figure 6. Simulation User Interface

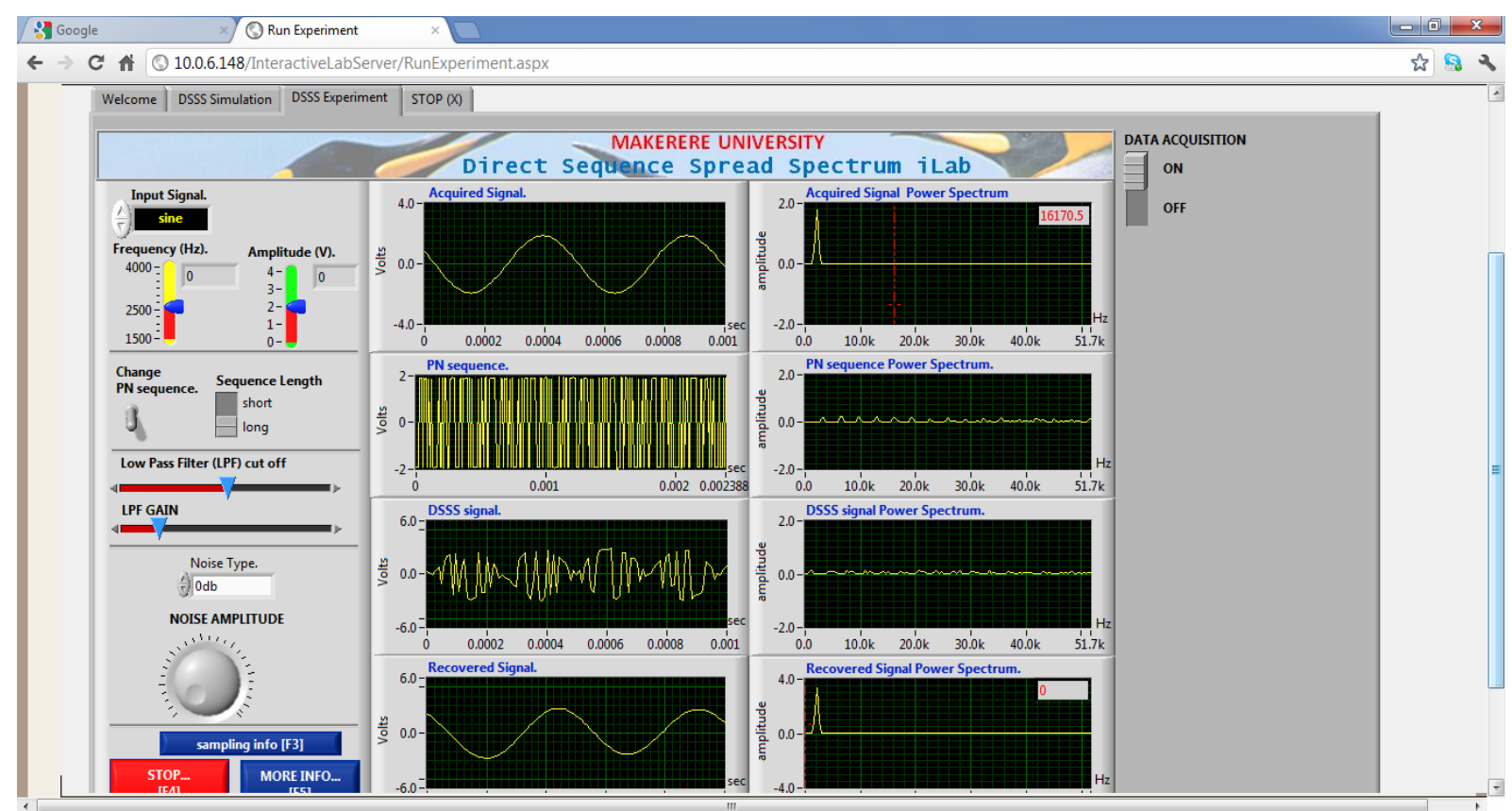

Figure 7. Data Acquisition Experiment User Interface 


\section{CONCLUSION}

This paper has presented a remote DSSS lab utilising the Emona DATEx. The lab will render support to curricula in the courses of Wireless Technologies and Mobile Communications Systems where there is currently no laboratory of any form available. In addition to improving the student pedagogical experience at MAK, the DSSS lab adds to the huge assortment of online laboratories available worldwide.

A voice synthesis VI implementation of the lab was developed but it wasn't deployed within the ISA due to non-support for audio rendering. This component would have improved the remote lab from standard monotone input signals to a real world voice alternative. Other future research considerations include; incorporating FHSS support and a wireless CDMA implementation as opposed to the current baseband version. This would clearly demonstrate other real world effects on spread spectrum communication such as multipath and the limitations and benefits posed by an increasing number of users on the communication channel.

\section{ACKNOWLEDGMENT}

An ounce of gratitude goes to the Government of the Republic of Uganda for the financial support to the research under the Presidential Innovations Fund. The authors are also indebted to the iLabs@MAK Project team, and the MIT iLabs team: Judson Harward, Kirky DeLong, Philip Bailey and James Hardison for the invaluable input during the implementation of the DSSS Lab. Special thanks also go to Lesley $\mathrm{Yu}$ of National Instruments, Alfred Breznik of EMONA Instruments and Paul Isaac Musasizi for their mentorship and technical guidance.

\section{REFERENCES}

[1] Roberto Boisson de Marca, "Trends in Wireless Communications". IEEE Communications Society, 2010

[2] Simon Haykin , "Communication Systems, 5th Edition", John Wiley \& Sons, 2009

[3] Valery P. Ipatov, "Spread Spectrum and CDMA: Principles and Applications", John Wiley \& Sons, 2005 http://dx.doi.org/ $\underline{10.1002 / 0470091800}$
[4] Andrea Goldsmith, "Wireless Communications", Cambridge University Press, 2008

[5] Cosmas Mwikirize, Arthur Tumusiime Asiimwe, Lea Musasizi, Victoria Namuswa, Mary Dawn Nakasozi, Charles Mugga, Andrew Katumba, Sandy Stevens Tickodri - Togboa, Julius Butime, Paul Isaac Musasizi., "Development of Online Laboratories for Modulation and Combinational Logic Circuit Analysis Using NI ELVIS II ${ }^{\mathrm{TM}}$ Platform", in Proceedings of Information Technology: New Generation - ITNG2010 Conference, 12th - 14th April 2010, Nevada, Las Vegas [In Transactions of IEEE/Computer Society].

[6] Cosmas Mwikirize, et al, "New Dimensions in Teaching Digital Electronics: A Multimode Laboratory Utilizing NI ELVIS IITM, LabVIEW and NI Multisim" in International Journal of Online Engineering (iJOE). Vol 6, No 4 (2010) pp. 49-54

[7] P. Nakazinga, P.Karumuna, A.T. Asiimwe, C. Mwikirize, P.I. Musasizi, S.S. Tickodri-Togboa, J. Butime and A. Katumba, "An Interactive System Modeling and Speed Control Online Laboratory Utilizing NI ELVIS II ${ }^{\mathrm{TM}}$ and the Quanser 010 Motor Control Trainer", presented at Remote Engineering and virtual Instrumentation (REV2011) Conference at Transylvania University, Brasov, Romania, June 28 - July 1, 2011

[8] EMONA INSTRUMENTS (2007), Emona Telecoms-Trainer ETT-202, Multi-Experiment Single Board Telecommunications Trainer for the popular NI ELVISTM Platform", retrieved on $7^{\text {th }}$ June 2010 from http://www.emona-datex.com

[9] James L. Hardison, K. D. (2008). "Deploying Interactive Remote Labs Using the iLab Shared Architecture". 38th ASEE/IEEE Frontiers in Education Conference, October 22 - 25, 2008 New York, Saratoga Springs

[10] National Instruments, (2010), "User Guide and Specifications: NI myDAQ", retrieved on 28th July 2011 from http://www.ni.com/ nimydaq/

[11] NI SCXI-1169. Retrieved from http://sine.ni.com /nips/cds/ view/p/lang/en/nid/14595 on 18th February 2012

\section{AUTHORS}

Helen Kyomugisha, Tom Kigezi, Cosmas Mwikirize, Roseline Akol, Doreen Orishaba, and Michael Kyesswa are with the College of Engineering, Design, Art and Technology, Makerere University, P.0. Box 7062, Kampala- Uganda (hkyomugisha@tech.mak.ac.ug, nkigezi@tech.mak.ac.ug, bmwikirize@tech.mak.ac.ug, rnakol@tech.mak.ac.ug,dorishaba@tech.mak.ac.ug, mkyesswa@tech.mak.ac.ug).

This article is an extended and modified version of a paper presented at the International Conference on Remote Engineering \& Virtual Instrumentation (REV2012), held at University of Deusto, Bilbao, Spain, July 4-6, 2012. Received 16 July 2012. Published as resubmitted by the authors 28 November 2012. 